\title{
SELEÇÃO DE FASES EM RELÉS NUMÉRICOS DE LINHAS DE TRANSMISSÃO BASEADA EM ANÁLISE MULTI-RESOLUÇÃO VIA TRANSFORMADA WAVELET
}

\author{
Paulo Márcio Silveira ${ }^{\dagger *}$ \\ Rui Seara* \\ pmarcio@iee.efei.br \\ Hans Helmut Zürn* \\ hans@labspot.ufsc.br \\ ${ }^{*}$ LINSE/LABSPOT - Departamento de Engenharia Elétrica, UFSC, \\ CEP: 88040-900, Florianópolis, SC, Brasil. \\ ${ }^{\dagger}$ GQEE/DET - Instituto de Engenharia Elétrica, Universidade Federal de Itajubá, UNIFEI \\ CEP: 37500-903, Itajubá, MG, Brasil.
}

\begin{abstract}
A new approach for fault type detection and identification in three-phase transmission lines is introduced. For this end, we have used the wavelet transform associated with a modal transformation. The wavelet coefficients, obtained by multiresolution decomposition, are then uncoupled. For short time analysis intervals, the energy levels in the transformed signals are extracted and compared with an appropriated threshold, determining then the fault type. A fault can be recognized in less than 2 ms after its occurrence. The Electromagnetic Transient Program (EMTP) and Alternative Transients Program (ATP) have been used for validating the obtained results.
\end{abstract}

KEYWORDS: Numeric Relays, Fault Identification, Pattern Recognition, Feature Extractor, Wavelet Transform.

\section{RESUMO}

Este artigo apresenta uma nova abordagem para a detecção e identificação de tipos de faltas em linhas de

Artigo submetido em 20/12/00

1a. Revisão em 26/02/02

Aceito sob recomendação do Ed. Assoc. Prof. Jacques Szczupak transmissão. O procedimento de identificação é baseado na transformada wavelet associada a uma transformação modal. Dessa forma, os coeficientes wavelet dos sinais de falta, obtidos por análise multi-resolução, são então desacoplados. Para curtos intervalos de análise, os níveis de energia contidos nos sinais transformados são extraídos e comparados a limiares apropriados, determinando-se o tipo de falta. Uma falta pode ser reconhecida em menos de $2 \mathrm{~ms}$ após sua ocorrência. $\mathrm{O}$ programa EMTP/ATP é utilizado para validar os resultados.

PALAVRAS-CHAVE: Relés Numéricos, Identificação de Faltas, Reconhecimento de Padrões, Extrator de Características, Transformada Wavelet.

\section{INTRODUÇÃO}

No contexto da proteção de linhas de transmissão, há diferentes tarefas que devem ser realizadas em tempo real, tais como: reconhecimento de faltas e seleção de fases, reconhecimento da direção de falta, estimação da localização de falta e bloqueio contra oscilação de potência.

Dentre as mais recentes abordagens para tais propósitos, podemos destacar as aplicações de sistemas especialistas 
(Kezunovic et alii, 1993), lógica fuzzy (Wang e Keerthipala, 1998) e redes neurais artificiais (Dalstein e Kulicke, 1995; Bo et alii, 1997; Coury e Jorge, 1998). Contudo, dependendo da tarefa de proteção bem como da complexidade do sistema a ser protegido, muito esforço computacional ainda é despendido, principalmente, na fase de pré-processamento. Tal esforço advém da necessidade de se extrair eficazmente as características relevantes dos sinais envolvidos, como também é decorrente da intensa fase de treinamento desses sistemas (principalmente quando dados brutos são utilizados). Além disso, o grande número de inferências, no caso de sistemas especialistas, ou ainda, as diversas conexões sinápticas, no caso das redes neurais, podem limitar ou mesmo inviabilizar essas técnicas sobretudo para operação em tempo real.

A identificação do tipo de falta objetivando a proteção de linha traz importantes benefícios, tais como: redução do tempo de computação para as outras tarefas do sistema de proteção, fornecimento de informações para o religamento automático monopolar e o chaveamento síncrono sob condições de falta.

Neste artigo, trataremos do problema de identificação de faltas. Para isso, introduzimos um novo método para seleção de fases a ser usado em relés de linhas de transmissão. Esse esquema apresenta um tempo de resposta (alguns milissegundos pós-falta) bastante curto quando comparado a outras abordagens. A razão do reconhecimento rápido se deve, principalmente, ao método empregado, ou seja: decomposição wavelet usando uma pequena janela de observação ( wavelet mãe de curta duração) associada a uma transformação modal.

De fato, a transformada wavelet (TW) tem demonstrado ser uma excelente extratora de características, superando algumas das dificuldades apresentadas por outros métodos. Essa ferramenta vem sendo utilizada com sucesso em diferentes áreas de aplicações em processamento de sinais. Ribeiro (1994) foi um dos primeiros a propor o uso da TW como ferramenta para análise de transitórios em sistemas de potência. Desde então, surgiram diferentes propostas de aplicação dessa ferramenta principalmente com objetivo de avaliar aspectos da qualidade da energia elétrica, destacando-se, nesse contexto, o trabalho de Santoso et alii (1996). Relacionado à área de proteção, poucas contribuições foram apresentadas até o momento, destacando-se: a detecção de faltas de alta impedância (Wai e Yibin, 1998) e a localização de falta (Magnago e Abur, 1997). Liang et alii (1998) propuseram um algoritmo de identificação de faltas baseado em análise multi-resolução. Apesar de sua relativa simplicidade, o algoritmo proposto por Liang et alii (1998) apresenta uma razoável habilidade para de- tectar e identificar faltas, inclusive aquelas com elevados valores de resistência. No entanto, o emprego de uma baixa taxa de amostragem associada à natureza seqüencial do algoritmo e à grande quantidade de comparações booleanas envolvidas pode demandar um tempo de processamento acima do esperado para o tratamento de determinados tipos de faltas. Além do mais, quando a corrente de carga na linha tende a ser da mesma ordem de grandeza das correntes de falta, métodos utilizando somente sinais de corrente levam o identificador a ficar confuso.

Em nossa proposta, os coeficientes wavelet são extraídos dos sinais trifásicos de tensão a fim de detectar súbitas mudanças que eventualmente possam ocorrer. Em seguida, esses coeficientes são desacoplados através de uma transformação modal e, finalmente, é realizada uma etapa de refinamento para enfatizar os respectivos padrões de faltas.

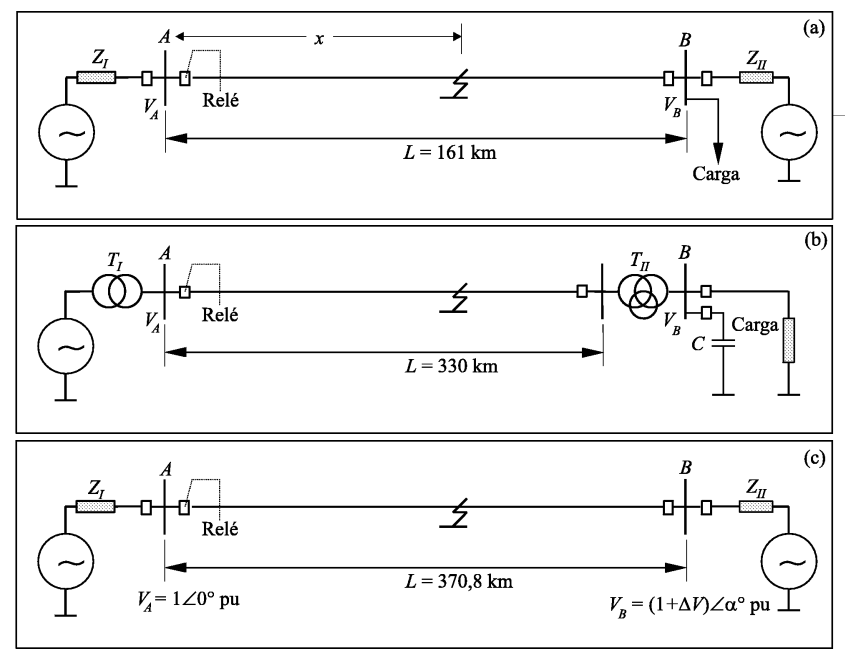

Figura 1: Linhas de Transmissão Simuladas. (a) Linha Transposta I (b) Linha Transposta II. (c) Linha não Transposta.

O presente artigo está estruturado da seguinte maneira: a Seção 2 apresenta um breve resumo da análise multiresolução (AMR) usando a TW, com o objetivo de dar suporte à metodologia utilizada; a Seção 3 apresenta uma descrição detalhada do método proposto; a Seção 4 mostra os resultados, considerando diferentes tipos de faltas, obtidos por simulações através dos programas EMTP/ATP, para os sistemas de transmissão mostrados na Figura 1 e, finalmente, a Seção 5 apresenta as conclusões e comentários finais. 


\section{ANÁLISE MULTI-RESOLUÇÃO}

A idéia básica da AMR é dividir o espectro de um sinal em sub-bandas e então tratar individualmente cada uma das sub-bandas considerando o propósito desejado. Quando a AMR é realizada através da transformada $w a-$ velet (Burrus et alii, 1998; Daubechies, 1992), é possível estabelecer um adequado compromisso entre as resoluções no tempo e na freqüência, permitindo uma eficiente extração de detalhes contidos nos sinais.

A análise wavelet parte da idéia de um espaço de funções de quadrado integráveis $L^{2}(\mathbf{R})$ e da conveniente escolha de uma função chamada função escalamento $\phi(x)$. Essa função é escolhida de modo a satisfazer algumas exigências, tais como: ter energia finita, ser oscilatória, ter média zero e um decaimento rápido em ambos os lados. Além dessas características, uma outra e mais importante, é a de que a família $\{\phi(x-k), k \in \mathbf{Z}\}$ deve formar uma base ortonormal para o subespaço de referência $V_{0}$. Assim,

$$
\cdots \subset V_{2} \subset V_{1} \subset V_{0} \subset V_{-1} \subset V_{-2} \cdots \subset L^{2}(\mathbf{R}) .
$$

Os subespaços $V_{j}$ podem ser vistos como uma seqüência de subespaços aninhados, tal que a $\cup_{j \in \mathbf{Z}} V_{j}$ seja densa em $L^{2}(\mathbf{R})$ e a $\cap_{j \in \mathbf{Z}} V_{j}$, vazia. Além disso,

$$
f(x) \in V_{j} \Leftrightarrow f(2 x) \in V_{j-1}, \quad j \in \mathbf{Z} .
$$

Deste modo, se o espaço $V_{j}$ é gerado por $\phi_{j, k}(x)$ para $j, k \in \mathbf{Z}$, então o espaço $V_{j-1}$ será gerado por $\phi_{j-1, k}(x)$ para $j, k \in \mathbf{Z}$. Portanto, $\phi_{j-1, k}(x)=\sqrt{2} \phi_{j, k}(2 x)$. Pelo fato de $V_{0} \subset V_{-1}$, qualquer função em $V_{0}$ pode ser descrita como uma combinação linear da função de base $V_{-1}$. Isso significa que $\phi(x)$ pode ser descrita em termos de uma soma ponderada de funções deslocadas $\phi(2 x)$, tal que

$$
\phi(x)=\sum_{k} g(k) \sqrt{2} \phi(2 x-k), \quad k \in \mathbf{Z},
$$

onde as amostras $g(k)$, definidas como o produto interno de $\phi(x)$ e $\sqrt{2} \phi(2 x-k)$, são os coeficientes do filtro de escalamento.

Considerando ainda o complemento ortogonal $\left(W_{j}\right)$ de $V_{j}$ para $V_{j-1}$, isto é, $V_{j-1}=V_{j} \oplus W_{j}$, pode-se definir:

$$
\psi(x)=\sum_{k}(-1)^{k} g(-k+1) \sqrt{2} \phi(2 x-k), \quad k \in \mathbf{Z},
$$

onde a função $\psi(x)$ é denominada wavelet mãe.

É importante ressaltar que a família de funções

$$
\psi_{j, k}(x)=2^{-j / 2} \psi\left(2^{-j} x-k\right), \quad j, k \in \mathbf{Z}
$$

forma uma base ortogonal para $L^{2}(\mathbf{R})$.

Desta forma, com um conjunto de funções $\phi_{k}(x)$ e $\psi_{j, k}(x)$ gerando todo o espaço $L^{2}(\mathbf{R})$, qualquer função $f \in L^{2}(\mathbf{R})$ pode ser descrita como uma expansão em série das funções wavelet e escalamento. Assim,

$$
f(x)=\sum_{k=-\infty}^{\infty} c_{\ell}(k) \phi_{\ell, k}(x)+\sum_{k=-\infty}^{\infty} \sum_{j=\ell}^{\infty} d_{j}(k) \psi_{j, k}(x) .
$$

Os parâmetros $d_{\ell}(k), d_{\ell+1}(k), d_{\ell+2}(k) \ldots$ são os coeficientes wavelet, e a seqüência $\left\{c_{\ell}(k)\right\}$ representa o sinal de mais baixa resolução em freqüência (coarsest scale) no nível $\ell$.

A TW, quando usada para analisar um sinal discreto, pode ser implementada usando-se dois filtros digitais: (i) um filtro passa-altas, com resposta ao impulso $h(n)$, relacionado a uma dada wavelet mãe $\psi(x)$; (ii) sua versão espelhada passa-baixas, com resposta ao impulso $g(n)$, associado à função escalamento $\phi(x)$. A relação entre $h(n)$ e $g(n)$ é dada por:

$$
h(n)=(-1)^{n} g(K-1-n), \quad n \in \mathbf{Z}
$$

onde $K$, considerado par, é o comprimento do filtro.

$\mathrm{Na}$ AMR, usando os filtros $h(n)$ e $g(n)$, é construída uma estrutura de filtragem para decompor (reconstruir) um sinal em diferentes níveis de resolução. Esse banco de filtros, também conhecido como decomposição piramidal, pode ser realizado considerando-se, por exemplo, três níveis de decomposição $(j=1,2,3)$, conforme mostrado na Figura 2. Nessa estrutura, $c_{0}(n)$ representa as amostras de $f(x) ; \quad c_{1}(n)$ é a versão suavizada (passa-baixas) e $d_{1}(n)$ é a versão detalhada (passa-altas) de $c_{0}(n)$. O próximo nível é baseado no sinal $c_{1}(n)$. Essa mesma lógica vai se repetir sucessivamente para as demais escalas de decomposição que forem necessárias.

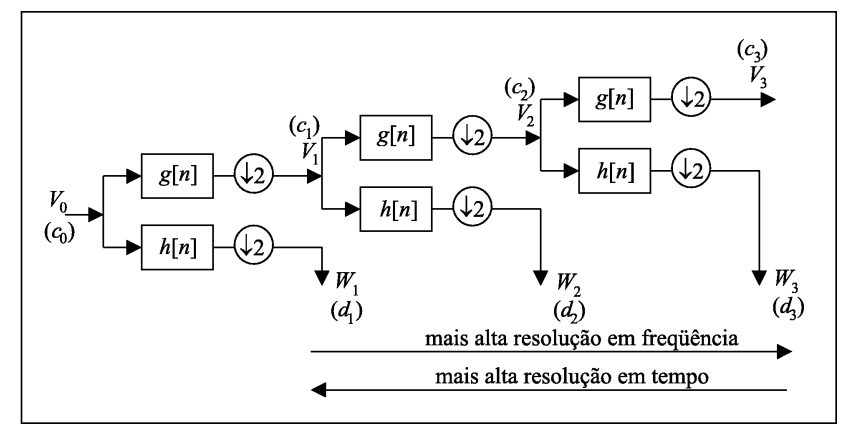

Figura 2: Estrutura de AMR Utilizando Três Níveis de Decomposição do Sinal.

A operação de dizimação por um fator dois, representada por $[\downarrow 2]$ nas saídas dos filtros $g(n)$ e $h(n)$, realiza um escalamento no sinal para o processamento do 
próximo nível de decomposição. Portanto, o sinal decomposto tem a metade das amostras do sinal anterior. Em aplicações que podem tolerar uma não unicidade, como no caso de detecção de transitórios, a operação de dizimação pode ser omitida, dando origem a uma transformada wavelet não dizimada (D'Attellis, 1997). Nesse caso, é necessário adaptar os filtros em função das escalas que serão utilizadas. Assim, para determinar os coeficientes $d_{j}$ e $c_{j}$, para $j=1,2,3, \ldots, N$, deve-se usar os filtros $h_{2}^{j}$ e $g_{2}^{j}$. Esses filtros são obtidos de $h(n)$ e $g(n)$, intercalando-se $2^{j-1}-1$ zeros entre cada um dos coeficientes desses filtros.

Embora seja possível utilizar mais níveis de decomposição para se construir um seletor de fases, somente os sinais de detalhamento na escala $1\left(d_{1}(n)\right)$ foram usados. Além disso, o uso de filtros wavelet do tipo Daubechies (1992) com quatro coeficientes (Db4), omitindo-se a operação de dizimação, tem-se mostrado suficiente para capturar eficazmente as informações relevantes para a identificação de todos os possíveis tipos de faltas.

\section{PROCEDIMENTO PROPOSTO}

Quando uma falta ocorre a uma distância $x$ da barra de alimentação de uma linha de transmissão (ver Figura 1(a)), os sinais de tensão e de corrente registrados nessa barra apresentam-se contaminados por ruídos de alta freqüência gerados durante a falta. Esses ruídos poderão ser de grande ou pequena amplitude, dependendo das características da falta, principalmente do ângulo de incidência e da distância de ocorrência do evento. Em qualquer dos casos, devido às características da AMR, a TW é bastante adequada para uma imediata detecção desses ruídos, até mesmo quando eles não são muito perceptíveis.

Para exemplificar tal afirmação, consideremos o caso do sinal de tensão apresentado na Figura 3(a), amostrado a $5 \mathrm{kHz}$. Esse sinal contém, além de um ruído branco, um outro ruído gerado durante um curto-circuito de alta impedância, e cujo ângulo de incidência ocorreu próximo da tensão zero volt. Esse distúrbio no sinal de tensão é praticamente inexistente quando observado a olho nu. Contudo, ao aplicar-se a decomposição wavelet, tal ruído é evidenciado logo na primeira saída que contém os detalhes do sinal, conforme mostra a Figura 3(b).

Deve-se considerar entretanto que, nas linhas de transmissão trifásicas, as fases são mutuamente acopladas e, por conseguinte, as perturbações de altas freqüências geradas durante uma falta podem aparecer nas fases não faltosas. Assim, apesar da excelente propriedade de detecção de faltas, a aplicação direta dos coeficientes wavelet para cada fase exigiria uma grande carga de processamento para extrair informações adequadas sobre as fases
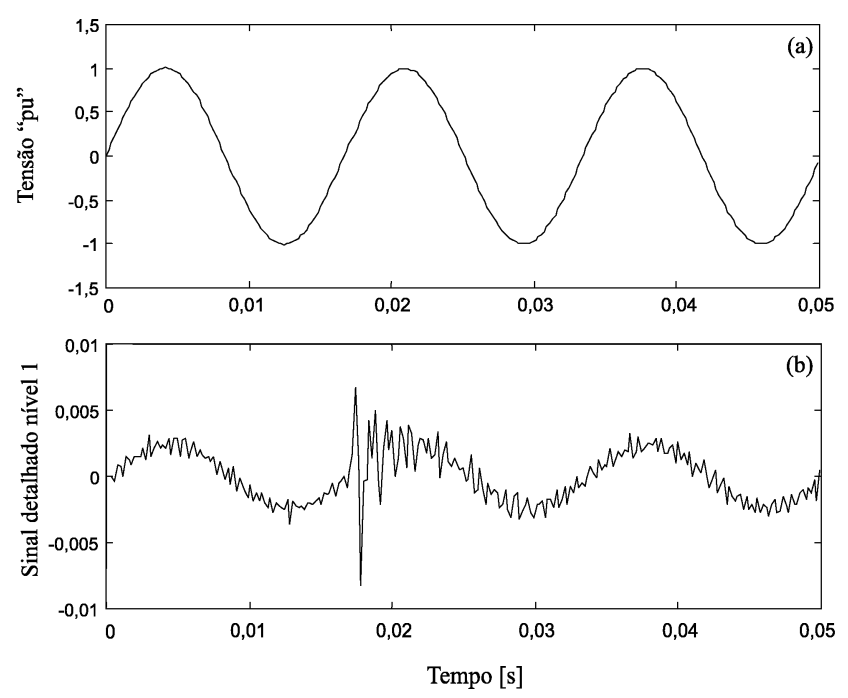

Figura 3: Detecção de Transitórios pela AMR. (a) Sinal Analisado. (b) Transitório Evidenciado.

faltosas. A Figura 4(a) ilustra esse fato, mostrando as formas de onda normalizadas de tensão, para uma falta fase A-terra ocorrida a $80 \%$ do comprimento da linha (Figura 1(a) sistema de $500 \mathrm{kV}$ ) com uma resistência de falta $\left(R_{f}\right)$ de $200 \Omega$. Além das características próprias da falta, um ruído branco foi adicionado, dando origem a um sinal cuja razão sinal-ruído (SNR) é de $60 \mathrm{~dB}$ sob condição normal de operação. Aplicando o filtro wavelet Db4, a cada uma das fases, obtêm-se os coeficientes wavelet nas várias escalas. Os coeficientes $d_{1}$ de cada fase são mostrados nas Figuras 4(b), 4(c) e 4(d). Embora, neste exemplo, seja até possível extrair as informações a respeito da falta (semelhança entre as seqüências $d_{1 B}$ e $d_{1 C}$ ), uma aplicação mais direta é desejada.

Por outro lado, utilizando-se uma apropriada transformação matemática, $n$ fases acopladas podem ser desacopladas obtendo-se $n$ circuitos independentes. Para isso, os sinais no domínio de fase devem ser decompostos em seus componentes modais por meio de uma transformação apropriada. As bem conhecidas transformações de Clarke (1943) (também conhecida como transformada $\alpha \beta 0$ ) e de Wedepohl (1963) não requerem uma linha de transmissão completamente transposta. Se existir um plano de simetria dos condutores em tal linha, os erros resultantes de suas aplicações são irrelevantes (Tavares et alii, 1999). Sendo assim, essas transformações foram adotadas, uma vez que os requisitos de suas aplicações são normalmente encontrados no mundo real.

O uso da transformação modal ajuda na identificação da falta, pois, dependendo do tipo de curto-circuito, alguns modos não são excitados, conforme ilustrado na Figura 5. Note que os nove sinais resultantes fo- 

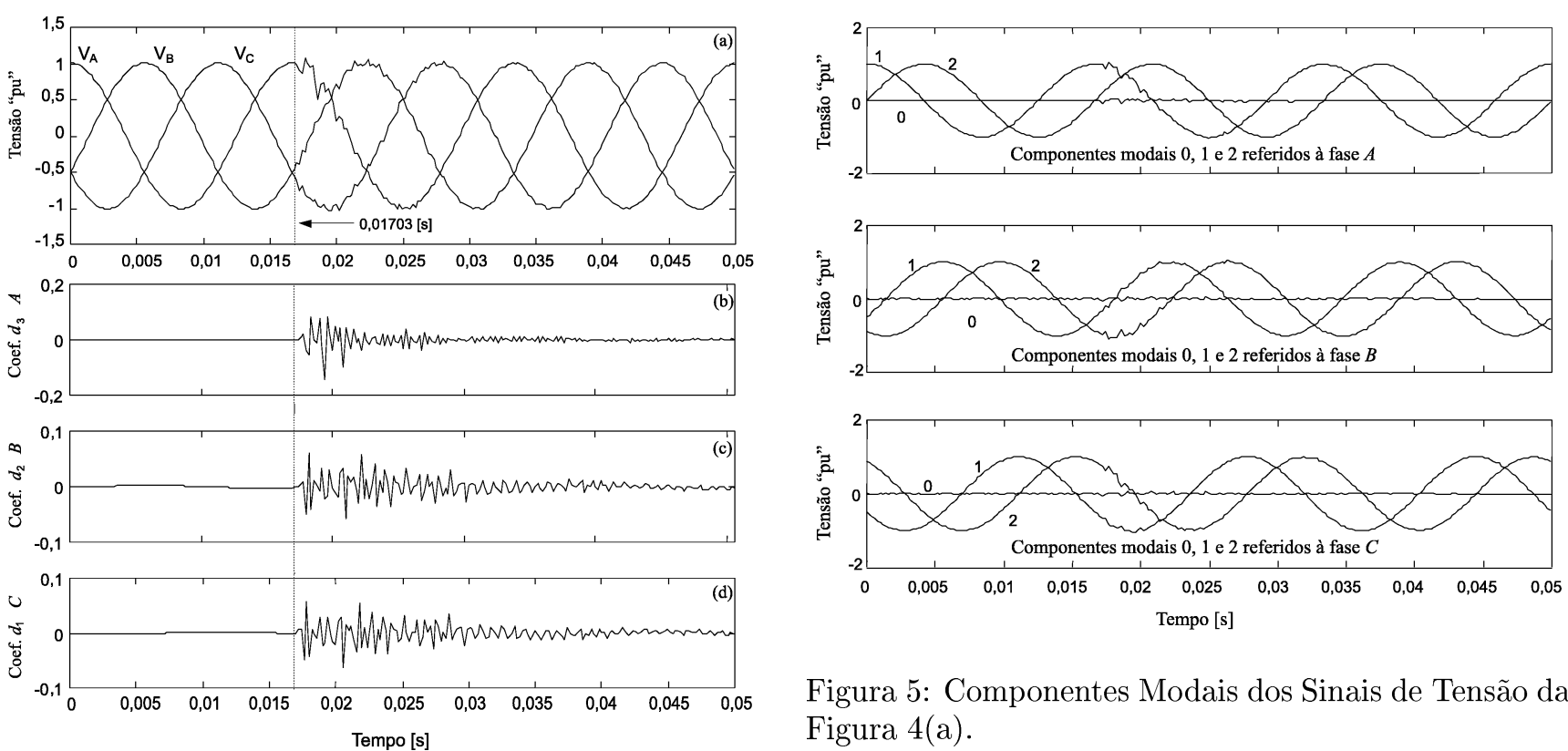

Figura 5: Componentes Modais dos Sinais de Tensão da Figura 4(a).

Figura 4: Sinais Mutuamente Acoplados. (a) Sinal Trifásico de Tensão. (b) Coeficientes Wavelet da Fase $A$, (c) da Fase $B$ e (d) da Fase $C$.

ram obtidos tomando, para cada transformação modal $(n \rightarrow n)$, uma das fases como referência, ou seja, $\left[V_{A}, V_{B}, V_{C}\right]^{T}, \quad\left[V_{B}, V_{C}, V_{A}\right]^{T}, \quad\left[V_{C}, V_{A}, V_{B}\right]^{T}$.

Desta forma, a aplicação da AMR aos sinais modais fornece valores muito pequenos de coeficientes wavelet para os modos não excitados (que denominaremos de modos de identificação) e valores significativos para outros modos. Essa característica é então utilizada para discriminar os diferentes tipos de faltas.

Como ambas as transformações - modal e wavelet - são operações lineares, torna-se possível efetuar primeiramente a filtragem wavelet, seguida pela transformação modal a fim de desacoplar os coeficientes resultantes. Essa seqüência de operações conduz a uma redução do número de operações executadas pelo algoritmo, visto que agora a filtragem wavelet é realizada apenas sobre os três sinais de fase e não mais sobre os nove sinais modais. Além do mais, como o modo terra é o mesmo para todos os sinais de referência $(A, B, C)$, esse modo pode ser calculado uma única vez. Do ponto de vista matemático, o procedimento descrito é representado por:

$$
\mathbf{d o}=\mathbf{W} \mathbf{d}_{1},
$$

onde $\mathbf{d}_{1}=\left[\begin{array}{lll}d_{1 A}, & d_{1 B}, & d_{1 C}\end{array}\right]^{T}$ e do $=$ $\left[\begin{array}{lll}d o_{1}, & d o_{2}, \ldots, & d o_{7}\end{array}\right]^{T}$ são os vetores de coeficientes wavelet acoplados e desacoplados, respectivamente. A matriz W (equação (9)) é denominada matriz de

pesos modais (Silveira et alii, 1999a, 1999b), que é uma versão modificada da matriz de transformação modal, no caso a transformação de Clarke (1943). Enfatiza-se aqui que a transformação representada por (8) deve ser efetuada a cada intervalo de amostragem.

$$
\mathbf{W}=\frac{1}{3}\left[\begin{array}{ccccccc}
1 & 2 & 0 & -1 & -\sqrt{3} & -1 & \sqrt{3} \\
1 & -1 & \sqrt{3} & 2 & 0 & -1 & -\sqrt{3} \\
1 & -1 & -\sqrt{3} & -1 & \sqrt{3} & 2 & 0
\end{array}\right]^{T}
$$

Desta maneira, o que se obteve foi um sistema que fornece sete saídas contendo os coeficientes wavelet dos sinais trifásicos de entrada, porém desacoplados, conforme ilustrado na Figura 6.

Resta agora enfatizar o exato momento da falta e extrair o padrão existente. Para tanto, tem-se utilizado o operador variância (ou dispersão) de um sinal. O conceito de variância está ligado à usual noção de energia $(\mathrm{AC})$ contida em um sinal $\left(W_{x}=\sum_{k}\left|x_{k}\right|^{2}\right)$. Para um sinal discreto $x(k)$, a variância é dada por:

$$
\sigma_{x}^{2}=E\left[(x(k)-\eta)^{2}\right]=\frac{\sum_{k=0}^{M-1}(x(k)-\eta)^{2}}{M-1}
$$

onde $\eta$ é o valor médio e $M$ é o comprimento da seqüência. Assim, pode-se obter a melhor estimativa não tendenciosa da variância se $x(k)$ for uma amostra de um processo gaussiano.

Deste modo, para se conseguir uma detecção precisa das faltas, procurou-se distribuir os "átomos de energia" uniformemente em (10), isto é, a variância $\left(\sigma_{l}^{2}\right)$ de cada sinal que representa um dos coeficientes wavelet desacoplados é calculada através de uma janela de dados 


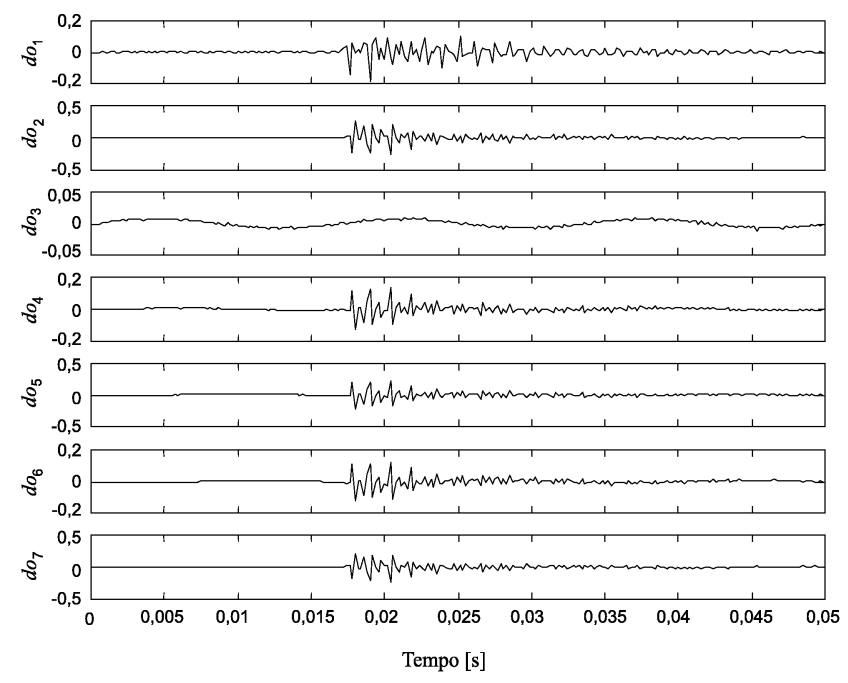

Figura 6: Coeficientes Wavelet Desacoplados.

móvel compreendendo $M$ amostras. Isso significa que, quando um novo coeficiente se torna disponível, o mais antigo dos $M$ coeficientes é descartado e um novo $\sigma_{l}^{2}$ é calculado como segue:

$$
\sigma_{l}^{2}(k)=\frac{\sum_{i=k-M+1}^{k}\left(d o_{l}(i)-\eta_{M}\right)^{2}}{M-1} .
$$

Para maior eficiência computacional, o cálculo de $\sigma_{l}^{2}$ pode ser executado somente nos intervalos inteiros $r$, com dependência do nível de decomposição, tal que $(r-1) H<k \leq r H$ para $r \in \mathbf{Z}$ e $H \in \mathbf{Z}^{+}$, ou seja:

$$
\sigma_{l}^{2}(r)=\frac{\sum_{k=(r-1) H+1}^{r H}\left(d o_{l}(k)-\eta_{M}\right)^{2}}{M-1}
$$

Para efeito de comparação de sinais, tem-se extraído a raiz quadrada de (12), obtendo-se o desvio padrão $\left(\sigma_{l}(r)\right)$ dos coeficientes desacoplados a cada janela móvel nos intervalos considerados.

Os valores de $M$ e $H$ devem ser suficientemente pequenos para os propósitos desejados (precisão e velocidade). Uma boa opção de projeto é fazer $M$ igual ao comprimento do filtro wavelet e $\mathrm{H}$ igual a $2^{j-1}$, sendo $j=1,2, \ldots, N$, o nível de decomposição.

Finalmente, os valores de $\sigma_{l}(r)$ são comparados a um limiar $(T)$ a fim de detectar o evento, isto é, cada saída é ativada sempre que

$$
\sigma_{l}(r) \geq T .
$$

Os valores de $T$ foram adotados com base em um levantamento do máximo valor de $\sigma_{l}(r)$ que pode ser encon-

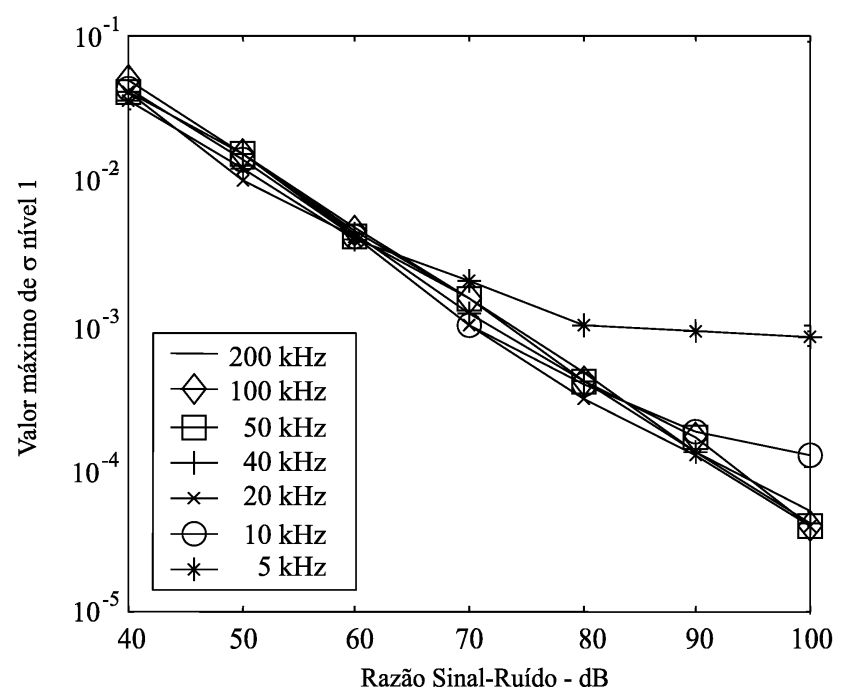

(a)

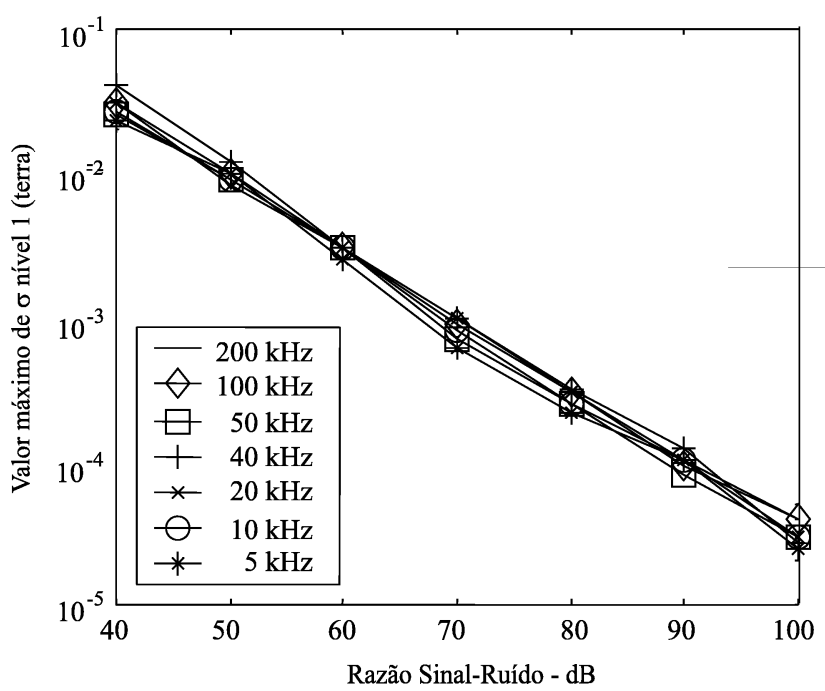

(b)

Figura 7: Valores Mínimos de Limiares para: (a) Saída 1, (b) Saídas de 2 a 7.

trado em regime permanente. Para isso, duas importantes características do sinal de entrada foram consideradas: o nível de ruído branco (razão sinal-ruído - SNR) e a freqüência de amostragem $\left(f_{s}\right)$. Como diferentes níveis de decomposição $(j)$ do sinal fornecem diferentes valores de $\sigma_{l}(r)$ em regime permanente, foi levado em consideração, no estudo dos limiares, outros níveis de decomposição, embora apenas o nível $j=1$ seja suficiente para os propósitos deste trabalho, conforme já observado. Além desses três fatores (SNR, $f_{s}$ e $j$ ), considerou-se também o limite máximo de variação do sinal de tensão nominal estabelecido por normas de operação, ou seja, $V_{\max }=1,1 V_{\text {nom }}$. 
Com base neste levantamento, estabeleceram-se os valores de limites mínimos que devem ser adotados como limiares, os quais foram dispostos em dois bancos de dados: um para a saída $S_{1}$ (modo aéreo) e outro para as demais saídas $S_{l} \quad(l=2$ a 7$)$. Os valores discretos contidos nesses bancos de dados podem ser representados por diferentes gráficos, como, por exemplo, os mostrados na Figura 7.

Assim, uma vez definida a freqüência de amostragem e o valor de SNR (com base em levantamento histórico ou por medição), os limiares ficam automaticamente definidos, considerando-se um certo fator de segurança. No caso de valores intermediários de freqüência de amostragem e de razão sinal-ruído, os limiares são obtidos utilizando-se interpolação linear.

A Tabela 1 apresenta os diferentes padrões que são produzidos nas saídas $\left(S_{l}, \quad l=1,2, \ldots, 7\right)$ do identificador para cada tipo de falta. O diagrama geral do identificador é ilustrado na Figura 8.

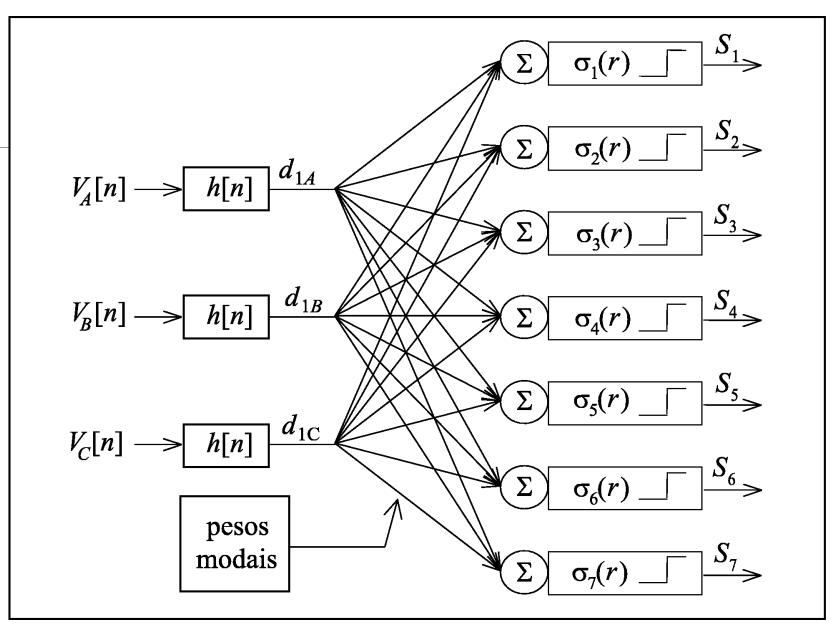

Figura 8: Diagrama de Blocos do Identificador de Faltas.

\section{RESULTADOS}

\subsection{Características dos dados simulados}

Os programas EMTP/ATP foram usados para simular diversas condições de faltas para os dois primeiros sistemas de transmissão representados nas Figuras 1(a) e 1(b). As linhas I e II são transpostas e modeladas como parâmetros distribuídos, e os dados (tensão e corrente) das três fases são obtidos na barra $A$. Vários tipos de faltas (fase-terra, fase-fase, fase-fase-terra, trifásica) em diferentes localizações ao longo da linha, com diferentes ângulos de incidência, resistências de falta e condições de carga têm sido considerados. Os sinais são amostrados a uma taxa de aproximadamente $5 \mathrm{kHz}$, e um nível de ruído branco é adicionado. Cada registro de falta é mantido em um banco de dados para posterior utilização durante os testes de desempenho do sistema de identificação.

\subsection{Faltas identificadas}

O identificador foi testado com aproximadamente 3200 diferentes faltas simuladas. Praticamente, $99 \%$ de todas as faltas foram efetivamente identificadas de acordo com a Tabela 1. Dentre as faltas consideradas não identificadas, muitas delas ainda apresentam um padrão possível de identificação, embora os sinais de saída não apresentem as características esperadas, tais como: transições simultâneas e intervalos de tempo no nível lógico "1" suficientes para a identificação. Tais características podem aumentar o tempo de identificação ou mesmo impossibilitar a identificação. Essas faltas são denominadas de faltas sutis, cujas características são: localização na outra extremidade da linha de transmissão, ângulo de incidência próximo da tensão zero com alta impedância $(\geq 200 \Omega)$ e elevada corrente de pré-falta. Não obstante, é necessário considerar que essas faltas simuladas com características muito peculiares raramente acontecem ou até mesmo podem ser consideradas irreais na prática, válidas apenas para propósitos de avaliação.

A Figura 9 mostra os sinais de saída do sistema identificador de faltas (utilizando a wavelet Db4) após a comparação com os limiares adotados (13). Nas entradas, foram aplicados os sinais de tensão apresentados na Figura $4(\mathrm{a})$.

Deve-se notar, na Figura 9, a indicação do exato instante em que as saídas assumem o nível lógico " 1 ". Considerando-se que o início do transitório ocorreu em 0,01703 ms, conforme indicado na Figura 4(a), o sistema levou $0,4 \mathrm{~ms}$ para que todas as saídas indicassem o correto padrão de falta $(A T)$. Considerando-se que a freqüência de amostragem é de $5 \mathrm{kHz}$, verifica-se que foram necessárias apenas duas amostras pós-falta para a ativação das saídas, em uma falta cujos transitórios não eram tão proeminentes. Isso enfatiza, portanto, a eficácia do método.

\subsection{Influência da não transposição das li- nhas}

A fim de avaliar o desempenho do algoritmo, nós o aplicamos a modelos de linha não transpostas com parâmetros dependentes da freqüência. Nesse caso, para a obtenção dos sinais de fase, foi usado o modelo de J. Marti (1982), cuja simplificação usual é tratar a matriz de transformação modal (própria para a linha simulada) como sendo real e independente da freqüência. Assim, 
Tabela 1: Padrão de Saída do Sistema Identificador

\begin{tabular}{|l|c|c|c|c|c|c|c|c|c|c|}
\hline & \multicolumn{10}{|c|}{ Tipo de Falta } \\
\hline$S_{l}$ & $A T$ & $B T$ & $C T$ & $A B$ & $B C$ & $C A$ & $A B T$ & $B C T$ & $C A T$ & $A B C$ \\
\hline$S_{1}$ & 1 & 1 & 1 & 0 & 0 & 0 & 1 & 1 & 1 & 0 \\
\hline$S_{2}$ & 1 & 1 & 1 & 1 & 0 & 1 & 1 & 1 & 1 & 1 \\
\hline$S_{3}$ & 0 & 1 & 1 & 1 & 1 & 1 & 1 & 1 & 1 & 1 \\
\hline$S_{4}$ & 1 & 1 & 1 & 1 & 1 & 0 & 1 & 1 & 1 & 1 \\
\hline$S_{5}$ & 1 & 0 & 1 & 1 & 1 & 1 & 1 & 1 & 1 & 1 \\
\hline$S_{6}$ & 1 & 1 & 1 & 0 & 1 & 1 & 1 & 1 & 1 & 1 \\
\hline$S_{7}$ & 1 & 1 & 0 & 1 & 1 & 1 & 1 & 1 & 1 & 1 \\
\hline
\end{tabular}
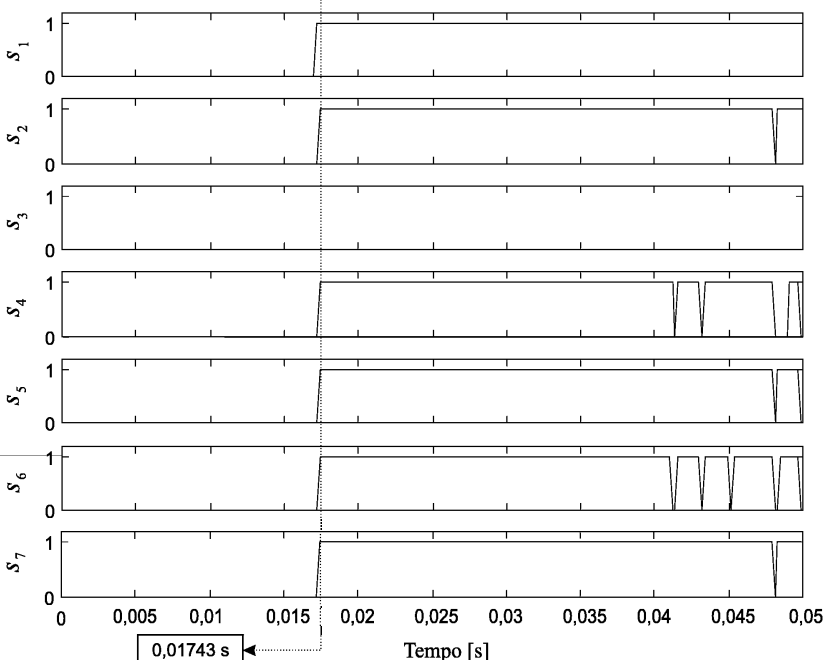

Figura 9: Saídas do Identificador de Faltas Considerando os Sinais de Entrada da Figura 4(a).

diferentes transitórios de falta foram obtidos simulandose a linha de transmissão que foi apresentada na Figura 1(c) e cujos principais dados para obtenção dos parâmetros de simulação estão ilustrados na Figura 10.

O principal aspecto a ser considerado em casos de linhas não transpostas é o fato de que a matriz de transformação modal separa os modos de propagação das ondas, porém não elimina os ruídos de falta em quaisquer dos modos. Em outras palavras, aparecerão ruídos de falta também naqueles modos que não são excitados como é o caso das linhas transpostas.

Contudo, resultados razoáveis ainda podem ser obtidos desde que os coeficientes nestas saídas (modos de identificação) sejam menores do que das outras saídas. Então, com um ajuste adequado de limiar, a falta pode ser identificada mesmo para um alto fator de desequilíbrio $(\mathrm{k} \cong 4 \%)$, como é o caso da linha considerada (GCOI, SCEL, 1997).

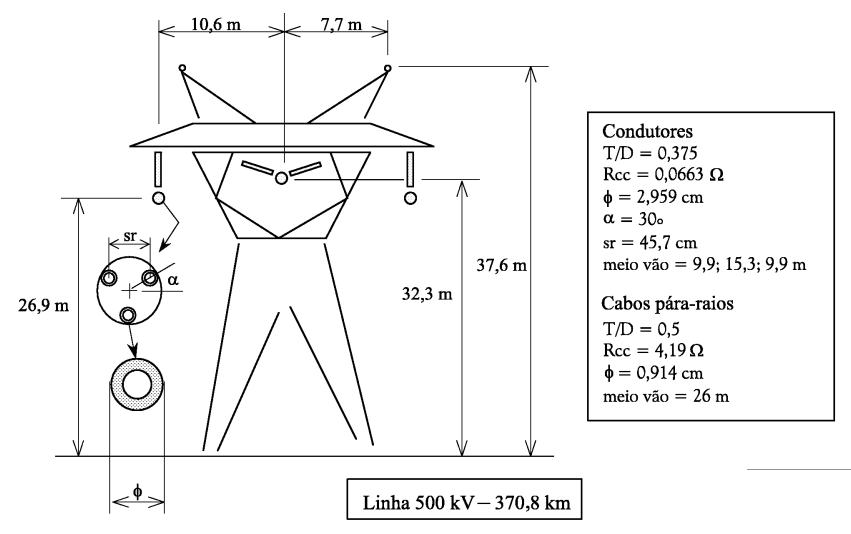

Figura 10: Modelo de Linha não Transposta.

Como exemplo, a Figura 11 mostra os coeficientes wavelet desacoplados para uma falta fase $C$-terra a $20 \%$ do comprimento da linha. Pode-se verificar que os coeficientes da saída 3 são menores do que os demais, indicando ainda uma falta $A T$.

$\mathrm{Na}$ prática, linhas longas devem ser transpostas por causa do alto fator de desequilíbrio nos sinais de tensão e corrente que elas podem causar. Mesmo assim, é importante salientar que nem sempre é possível uma completa e perfeita transposição, e um certo nível de desequilíbrio existirá. Esses desequilíbrios, embora pequenos e dentro dos critérios estabelecidos pelas normas de operação das linhas de transmissão - valores até 1,6\% são aceitáveis conforme (ONS, 2000)-, também foram considerados nas simulações e, nesses casos, o algoritmo apresentou desempenho similar ao das linhas perfeitamente transpostas.

\subsection{Tempo de resposta}

O tempo de resposta $\left(t_{r}\right)$ do identificador de faltas pode ser calculado por:

$$
t_{r}=\frac{1}{f_{s}} N a+t_{e}
$$




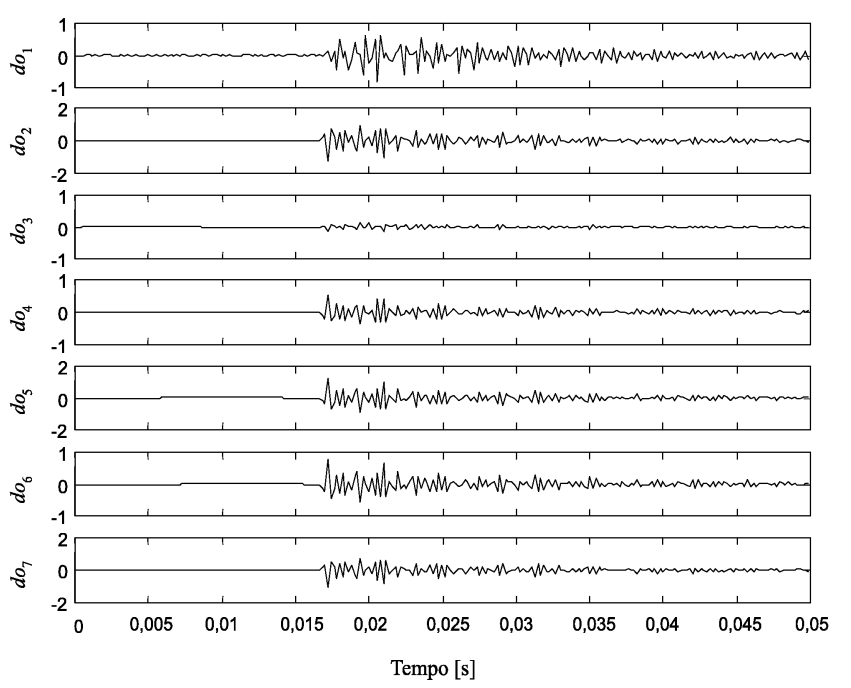

Figura 11: Coeficientes Wavelet Desacoplados para a Linha não Transposta.

onde $f_{s}$ é a freqüência de amostragem, $N a$ é o número de amostras até a ocorrência da transição $(0 \rightarrow 1)$ em todas as saídas e $t_{e}$ é o tempo necessário para a execução do algoritmo. Embora o tempo de execução $\left(t_{e}\right)$ ainda não tenha sido avaliado, os autores têm considerado o poder dos atuais processadores de sinais digitais e recentes desenvolvimentos em transdutores ópticos de tensão e corrente, habilitando registros e tratamento com altas taxas de amostragem. Também são consideradas as recentes pesquisas em processamento paralelo diminuindo muito os tempos de processamento. Assim, não levando em conta o tempo de execução do algoritmo de identificação $\left(t_{e}\right)$, o tempo de resposta estimado do identificador é menor do que $2 \mathrm{~ms}$, uma vez que, para a grande maioria das faltas, $N a$ tem variado entre 1 e 8 amostras pós-falta, para $f_{s}=5 \mathrm{kHz}$.

\subsection{Desafios e limitações}

Todas as faltas simuladas também foram identificadas utilizando-se os sinais de corrente. Em alguns casos, o uso dos sinais de corrente associados aos sinais de tensão pode auxiliar a identificação das faltas, embora a melhoria não tenha sido significativa para muitos dos casos observados.

Faltas com elevado valor de curto-circuito em relação ao valor nominal podem ter padrões de identificação melhorados para freqüências de amostragem menores do que a adotada, entretanto com maior tempo de identificação. Além do mais, quando a corrente de pré-falta é elevada, o identificador não apresenta bons resultados para o caso de faltas com alta impedância.
A AMR é bastante sensível ao ruído não correlacionado. Assim, para faltas sutis (quase sem transitório), o procedimento proposto não obteve sucesso para valores de SNR abaixo de $60 \mathrm{~dB}$. É óbvio que, nesses casos, a perturbação gerada pela falta estará no mesmo patamar do nível de ruído, não sendo mais possível distinguir o instante do distúrbio. Investigações nessa direção estão em andamento considerando outros níveis de decomposição e outros tipos de wavelets.

A grande maioria de faltas em linhas de transmissão é do tipo fase-terra. Em muitas companhias de energia elétrica que adotam a filosofia de religamento monopolar, a prática comum é desligar todas as três fases para as faltas que não sejam do tipo fase-terra. Assim, tornamse suficientes os padrões apresentados na Tabela 1 para o caso de faltas fase-fase-terra, embora as fases envolvidas não tenham sido identificadas. No entanto, tem-se trabalhado para identificar as fases nesse tipo de ocorrência, contribuindo-se também para outras tarefas de proteção.

\subsection{Melhorias}

Resultados mostram que, ao se trabalhar com altas freqüências de amostragem (50 a $200 \mathrm{kHz}$ ), a AMR possibilita a captura de frentes de ondas viajantes, as quais irão fornecer além da identificação do tipo de falta, incluindo a fase-fase-terra, também a distância da mesma.

Tal abordagem foi recentemente finalizada e resultados da pesquisa serão brevemente apresentados à comunidade científica da área.

\section{CONCLUSÕES}

Este artigo propõe uma metodologia para a identificação do tipo de falta em linhas de transmissão, utilizando a transformada wavelet. Os resultados obtidos são encorajadores e a técnica proposta requer um esforço computacional relativamente baixo. Portanto, essa técnica pode ser usada como parte de um algoritmo em relés numéricos de alta velocidade ou mesmo em dispositivos para diagnóstico de qualidade da energia. Pelos estudos iniciais, acredita-se que os algoritmos baseados em TW têm um enorme potencial para fazer parte de uma nova geração de relés de proteção e dispositivos de medição. Pesquisas adicionais estão sendo empreendidas para estender a aplicação mencionada a diferentes modelos de sistemas de transmissão, tais como: linhas multiterminais e linhas de circuitos paralelos com alto fator de desequilíbrio; além de outras tarefas de proteção.

\subsection{Agradecimentos}

Os autores agradecem o apoio financeiro da CAPES. 


\section{REFERÊNCIAS}

Bo, Z. Q., R. K. Aggarwal, A. T. Johns, H. Y. Li and Y. H. Song (1997). "A New Approach to Phase Selection Using Fault Generated High Frequency Noise and Neural Networks". IEEE Trans. PWRD, Vol.12, No. 1, January, pp. 106-115.

Burrus, C. S., R. A. Gopinath and H. Guo (1998). Introduction to Wavelets and Wavelet Transforms. Prentice-Hall, New Jersey.

Clarke, E. (1943). Circuit Analysis of AC Power System. Vol. I, John Wiley \& Sons, New York, 1943.

Coury, D. V. \& D. C. Jorge (1998). "Artificial Neural Network Approach to Distance Protection of Transmission Lines". IEEE Trans. PWDR, Vol. 13, No. 1, January, pp. 102-108.

D'Attellis, C. E., and E. M. Fernández-Berdaguer (1997). Wavelet Theory and Harmonic Analysis in Applied Sciences. Birkhäuser Boston.

Dalstein, T. \& B. Kulicke (1995). "Neural Network Approach to Fault Classification for High Speed Protective Relaying". IEEE Trans. PWRD, Vol. 10, No. 2, April, pp. 1002-1011.

Daubechies, I., (1992). Ten Lectures on Wavelets. SIAM, Philadelphia, Pennsylvania.

Eletromagnetic Transient Program - EMTP (1992) Rule Book and User's Guide, Eletrotek Concepts INC.

GCOI/SCEL e GCPS/CTST (1997). Procedimentos de Medição para Aferição de Qualidade de Ondas de Tensão quanto ao Aspecto de Conformidade (Distorção Harmônica, Flutuação e Desequilíbrio de Tensão). Normas, Brasília, Brasil.

Kezunovic, M., P. Spasojevic, C. W. Fromen and D. R. Sevick (1993). "An Expert System for Transmission Substation Event Analysis". IEEE Trans. PWRD, Vol. 8, No. 4, October, pp. 1942-1949.

Liang, J., S. Elangovan and J. B. X. Devotta. (1998). "A Wavelet Multiresolution Analysis Approach to Fault Detection and Classification in Transmission Lines". Electric Power \& Energy Systems, Vol. 20, No. 5, pp. 327-332.

Magnago, F. H. \& A. Abur (1998). "Fault Location Using Wavelets". IEEE Trans. PWRD, Vol. 13, No. 4, October, pp. 1475-1480.
Martí, J. R. (1982). Accurate Modelling of FrequencyDependent Transmission Lines in Electromagnetic Transients Simulations. IEEE Trans. PAS, Vol. 101, No. 1, January, pp. 147-157.

ONS - Operador Nacional do Sistema (2000). Requisitos Mínimos para Linhas de Transmissão. Procedimentos de Rede, Sub-módulo 2.4, Brasília, Brasil.

Ribeiro, P. F. (1994). "Wavelet Transform: An Advanced Tool for Analyzing Non-Stationary Harmonic Distortions in Power Systems". Proceedings IEEE ICHPS VI, Bologna, Itália, pp. 365-369.

Santoso, S., E. J. Powers, W. M. Grady and P. Hofmann (1996). "Power Quality Assessment Via Wavelet Transform Analysis". IEEE Trans. PWRD, Vol.11, No. 2, April, pp. 924-930.

Silveira, P. M., R. Seara and H. H. Zürn (1999a). "Fault Type Identification Using Wavelet Transform". The International Conference on Intelligent Systems Application to Power Systems - ISAP'99, pp. 267-272, Rio de Janeiro, Brasil.

Silveira, P. M., R. Seara and H. H. Zürn (1999b). "An Approach Using Wavelet Transform for Fault Type Identification in Digital Relaying". IEEE PES Summer Meeting 1999, pp. 937-942, Edmonton, Canadá.

Tavares, M. C., J. Pissolato, C. M. Portela (1999). Quasi-Modes Three-Phase Transmission Line Model - Comparison With Existing Frequency Dependent Models. IPST'g9 - International Conference on Power Systems Transients, Budapest, Hungary, June, pp. 49-54.

Wai, D. C. T. \& X. Yibin (1998). "A Novel Technique for High Impedance Fault Identification". IEEE Trans. PWRD, Vol.13, July, No. 3, pp. 738-744.

Wang, H. and W. W. L. Keerthipala (1998). "FuzzyNeuro Approach to Fault Classification for Transmission Line Protection". IEEE Trans. PWRD, Vol. 13, No. 4, October, pp. 1093-1102.

Wedepohl, L. M. (1963). "Application of Matrix Methods to the Solutions of Travelling-Wave Phenomena in Polyphase Systems". Proc. of IEE, Vol. 110, No. 12, December, pp. 2200-2212. 